\title{
Programmer's Guide to the 1996 Demo Executor
}

\section{Flater \\ Evan Walloee}

U.S. DEPARTMENT OF COMMERCE Technology Administration National Institute of Standards and Technology

Gaithersburg, MD 20899-0001

ac

100

.456 



\section{Programmer's Guide to the 1996 Demo Executor}

\section{Flater}

U.S. DEPARTMENT OF COMMERCE Technology Administration

National Institute of Standards and Technology

Gaithersburg, MD 20899-0001

February 1997

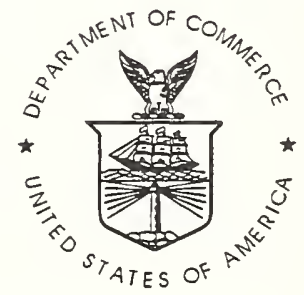

U.S. DEPARTMENT OF COMMERCE William M. Daley, Secretary

TECHNOLOGY ADMINISTRATION

Mary L. Good, Under Secretary for Technology

NATIONAL INSTITUTE OF STANDARDS

AND TECHNOLOGY

Arati Prabhakar, Director 


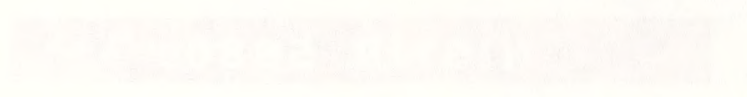




\section{Programmer's Guide to the 1996 Demo Executor}

\section{Contents}

1 Introduction $\quad 1$

2 The Executor $\quad 2$

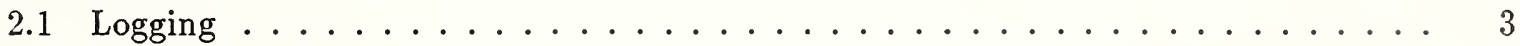

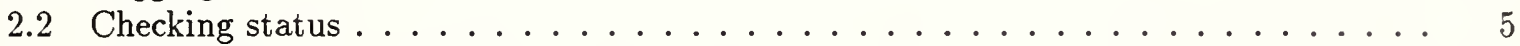

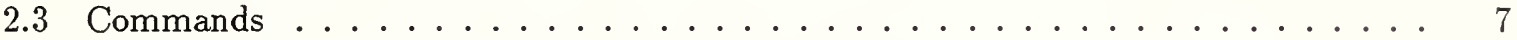

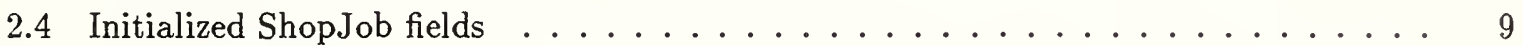

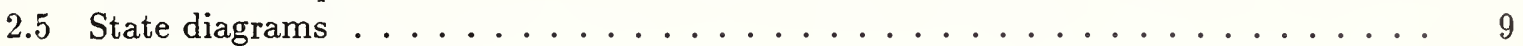

3 Issues for Future Work $\quad 9$

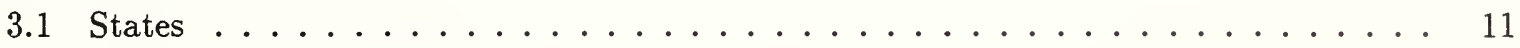

3.2 Job control commands . . . . . . . . . . . . . . . . 11

4 Further Reading $\quad 12$

$\begin{array}{ll}\text { A job_mgr.idl } & 13\end{array}$

$\begin{array}{ll}\text { B executor.idl } & 17\end{array}$

$\begin{array}{lr}\text { C workcell.idl } & 19\end{array}$

\section{List of Figures}

1 Architecture of the NAMT Framework Demo . . . . . . . . . . . . . . . 1

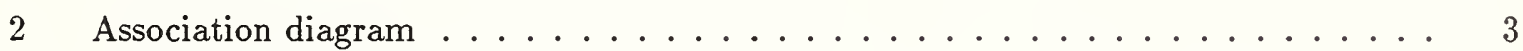

3 Guardian screen showing $\operatorname{logs} \ldots \ldots \ldots \ldots \ldots \ldots$

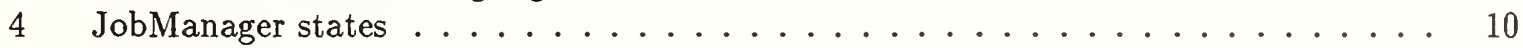

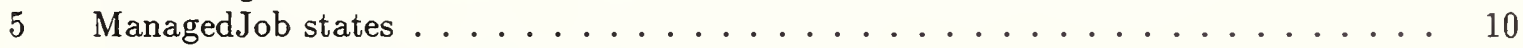

6 Proposed ManagedJob states (concurrent model) . . . . . . . . . . . . . 11

\section{List of Tables}

1 Status operations available on the Executor ................ 5

2 Status operations available on all ManagedJobs ................ . 6

3 Additional ShopJob status operations ................... 6

4 Additional ShopProcessJob status operations . . . . . . . . . . . . . 6

5 Commands affecting the state of the Executor . . . . . . . . . . . . . . 7

6 Commands affecting one shop-level job . . . . . . . . . . . . . . 7

7 Commands affecting all shop-level jobs . . . . . . . . . . . . . 8

This work was funded through the National Advanced Manufacturing Testbed (NAMT) project and the Systems Integration for Manufacturing Applications (SIMA) program. 



\title{
Programmer's Guide to the 1996 Demo Executor
}

\author{
David Flater \\ Evan Wallace
}

March 6, 1997

\begin{abstract}
This report documents the interfaces and functions of the 1996 demo version of the Executor. The Executor is the shop controller for the National Advanced Manufacturing Testbed (NAMT) Framework demo, a prototype distributed manufacturing system that is used to validate prenormative specifications for manufacturing-related protocols and interfaces.
\end{abstract}

\section{Introduction}

The National Advanced Manufacturing Testbed (NAMT) Framework[1] demo is a prototype distributed manufacturing system that serves as a testbed and trial implementation for emerging industry-developed specifications such as SEMATECH's Computer Integrated Manufacturing (CIM) Framework. [2] The core of the NAMT Framework demo is the Executor, which acts as the shop controller in the NAMT Framework architecture (see Figure 1).

The Executor is a multi-threaded Common Object Request Broker Architecture (CORBA) server. It dispatches discrete parts manufacturing jobs that are requested by a human operator via the Guardian. The Guardian is the user interface that accepts input from a terminal and translates it into CORBA commands understood by the Executor. Other components in the architecture are the Production Information Base (PIB), which uses a commercial object base to maintain lot information; the Product Data Manager (PDM), which uses another commercial product to maintain STEP (Standard for the Exchange of Product model data) Part 21 documents containing the routings for the lots; and the workcell, which is the next level of control down from the Executor, closer to the actual machines.

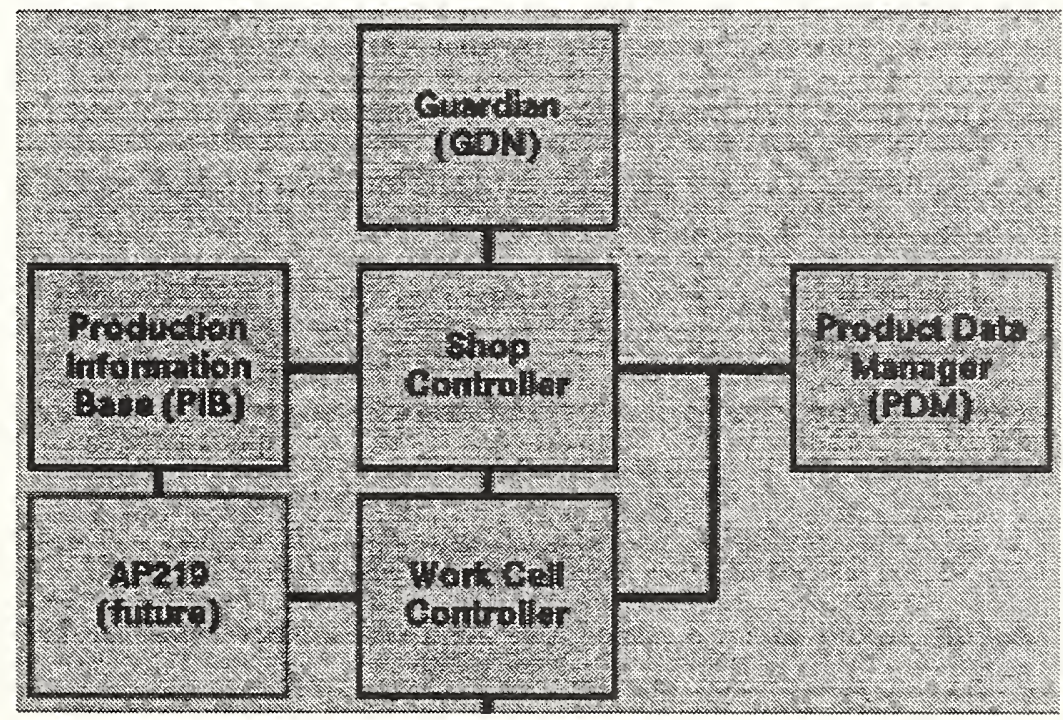

Figure 1: Architecture of the NAMT Framework Demo 
This guide to the Executor is written at a level to be understood by a programmer, rather than a casual user, because the Executor itself does not interface directly with the user. It is the Guardian's job to provide user-friendly access to the Executor's functions, and to mediate between the keyboard and mouse on one hand and the Executor's CORBA API on the other. The purpose of this report is to document that API as it is seen by the Guardian or by any other clients of the Executor that may be built in the future. Documentation at an even lower level that discusses the internals of the Executor and various implementation issues is available on the NIST intranet at <URL:http://www-i.cme.nist.gov/framework/doc/papers96/ex96docs/>.

\section{The Executor}

The primary functions of the Executor are the following:

1. Respond to commands and status requests from the Guardian, which receives control input directly from the operator.

2. Retrieve lot information from the PIB to determine the routings.

3. Retrieve routings from the PDM and parse them.

4. Activate jobs.

5. Dispatch tasks to workcells.

6. Receive feedback from the workcells.

7. Abort jobs that are botched.

8. Write logging information.

The job control and status monitoring interfaces that the Executor offers to the Guardian are documented in following sections. The interface provided by the workcell to the Executor is largely the same as that provided by the Executor to the Guardian; both inherit the primary aspects of their interface from the same class. The other interfaces supported by the Executor, namely those to the PIB and the PDM, are out of the scope of this report, since they are defined unilaterally by the PIB and PDM; however, the interactions with the PIB and PDM are important to understanding how the Executor works.

In order to show the Executor in context, the following scenario describes its interactions with the Guardian, the PIB, the PDM, and the workcell by following the path of a job through the system. Figure 2 illustrates the associations between the various entities that will be discussed below.

- The Guardian asks the Executor to create a new shop-level job.

- The Guardian supplies values for the empty fields in the shop job (job name, lot, and priority), then informs the Executor that the job is ready to go.

- The Executor gets the lot ID from the job, retrieves the lot info from the PIB, and extracts the ID of the routing from the lot.

- The Executor retrieves the routing info (a STEP Part 21 document) from the PDM and parses it.

- The Executor constructs a list of ShopProcessJobs corresponding to the tasks in the routing and makes note of the workcells that are eligible to perform each one.

- When the shop has resources for another job, the Executor activates the job.

- When a workcell becomes available for the first task in the job, the Executor starts the task in the same way that the Guardian started the shop-level job: by asking the workcell to create a new job, filling in the fields, and then asking the workcell to start it. 


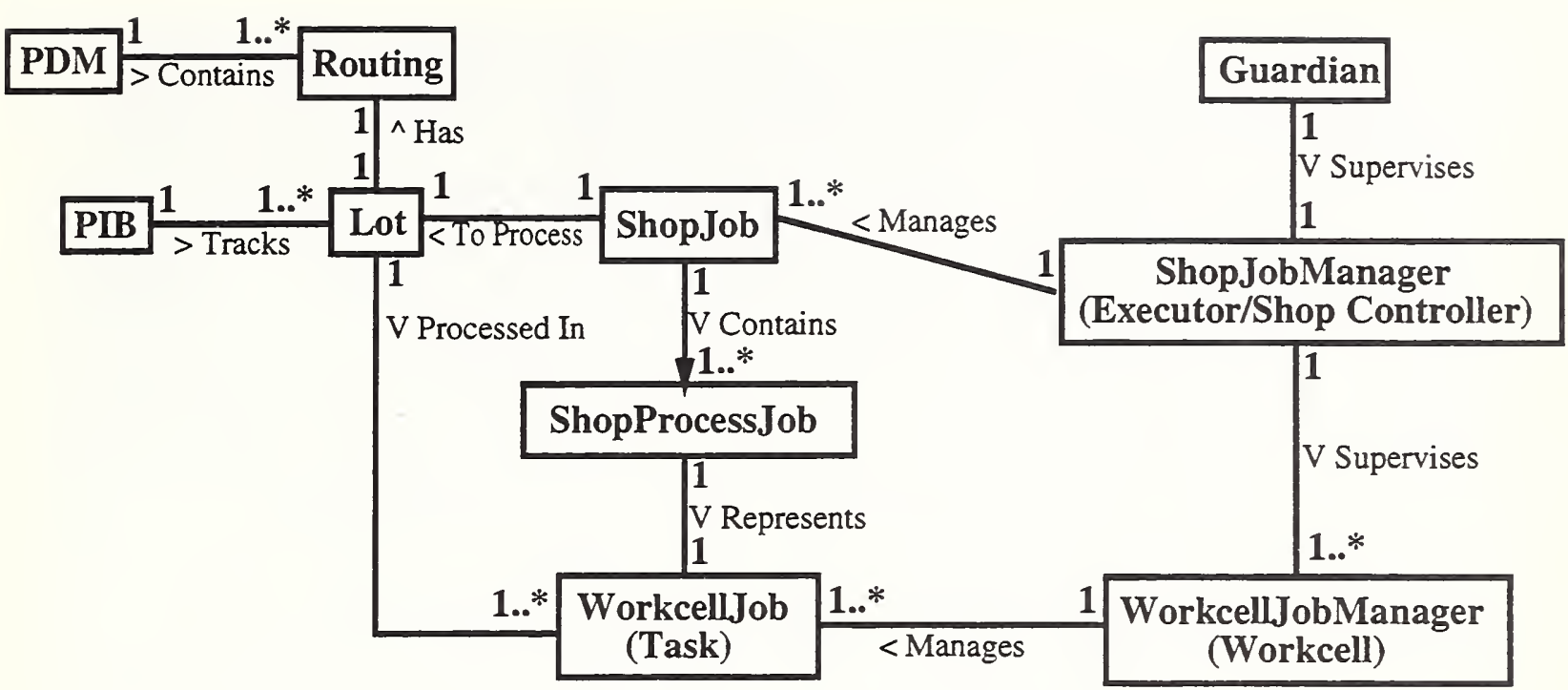

Figure 2: Association diagram

- The workcell performs the task, then informs the Executor when it is done. If the task is botched, the workcell informs the Executor of this too, so that the Executor can take appropriate action.

- The Executor recalculates the state of the shop-level job (e.g. Executing, Completed, Stopped, or Aborted) from the states of its constituent tasks. If the job is still Executing, the Executor proceeds to dispatch its next task.

In addition to the activity described above, the Executor is continuously polled for status information, and logs are maintained.

The Executor's job control and status monitoring interfaces are defined in IDL, the Interface Definition Language that is used for CORBA servers. Those IDL files are provided in the appendices and are explained in detail in following sections. The class hierarchy is very simple:

- JobManager: generic superclass for Executor and workcell controllers.

- ShopJobManager: the class of the Executor.

- WorkcellJobManager: the class of the workcell controller.

- ManagedJob: generic superclass for ShopJob, ShopProcessJob, and WorkcellJob.

- ShopJob: the class of a shop-level job.

- ShopProcessJob: the class that the Executor uses to keep record of workcell tasks.

- WorkcellJob: the class of a workcell task, owned by the workcell.

\subsection{Logging}

The Executor maintains three of the four logs that are displayed by the Guardian (see Figure 3); two directly, and one indirectly via the PIB client library. Logging is used to track the nature of the message traffic between the Executor and the other components. The lines of text are color-coded to indicate which specification was the source for each message.

The format for a line in a log file is "KEYWORD text..." with KEYWORD being one of the following choices to indicate the source specification:

- AP203: STEP Part 203, Configuration Controlled Design 


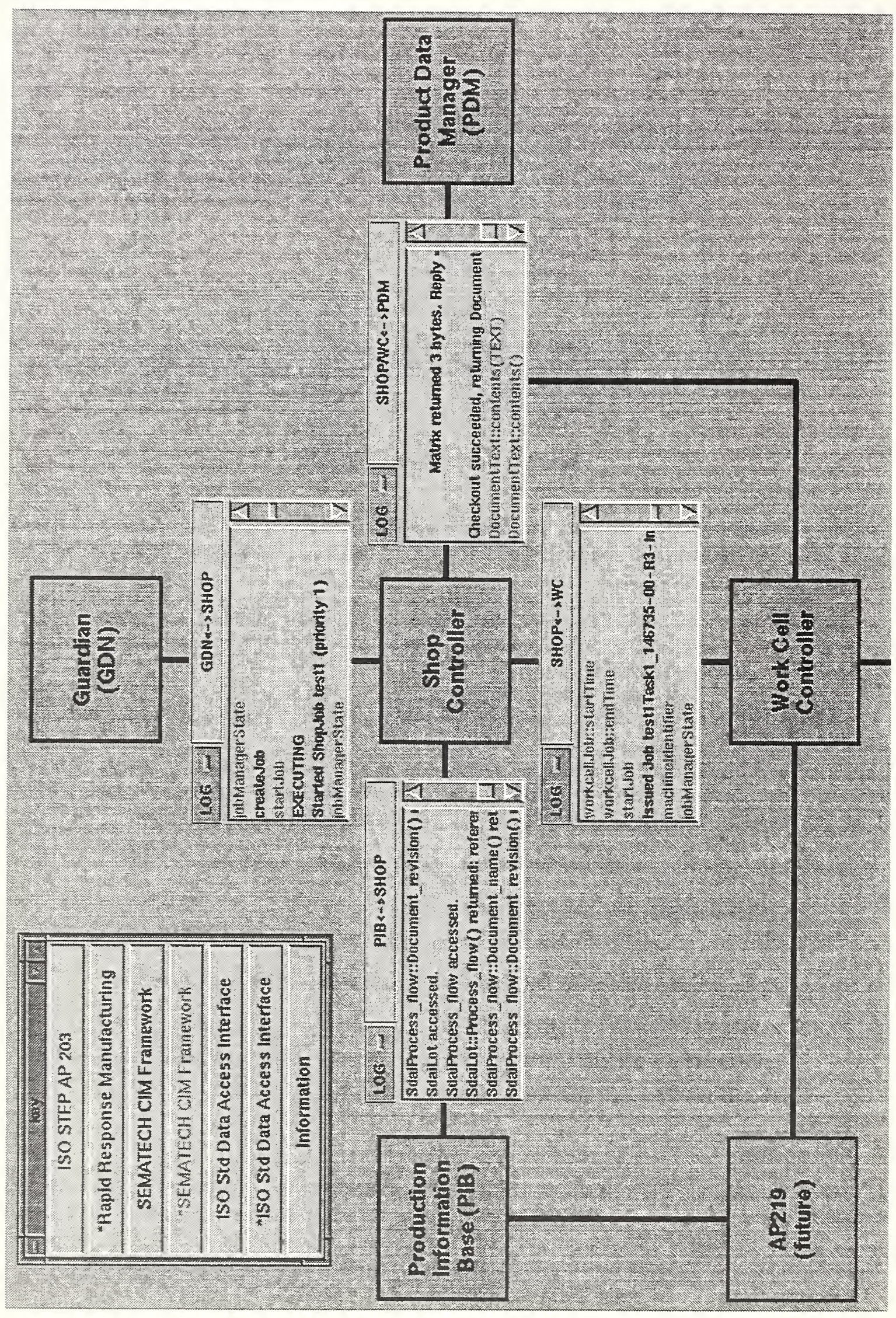

Figure 3: Guardian screen showing logs 
- RRM*: Modified Rapid Response Manufacturing (RRM) for PDM/IDL

- CIMF: SEMATECH CIM Application Framework (CIMF)

- CIMF*: Modified CIMF

- SDAI: Standard Data Access Interface (SDAI)

- SDAI*: Modified SDAI

- INFO: Not a message, just useful information

\subsection{Checking status}

The Executor provides a set of top-level operations by which a client can check the Executor's status and get handles to shop jobs and workcells (Table 1). The client can get more information on jobs and workcells by calling operations on them directly once a handle has been retrieved. Table 2 shows the status operations provided by all ManagedJobs, while Tables 3 and 4 show the additional specialized status operations provided by ShopJobs and ShopProcessJobs respectively.

Table 1: Status operations available on the Executor

\begin{tabular}{|l|l|}
\hline \multicolumn{1}{|c|}{ Operation } & \multicolumn{1}{|c|}{ Description } \\
\hline boolean isPaused(); & $\begin{array}{l}\text { Returns true if the Executor is in the } \\
\text { Paused state. }\end{array}$ \\
\hline jm_state jobManagerState(); & $\begin{array}{l}\text { Returns the state of the Executor as } \\
\text { an enum. }\end{array}$ \\
\hline $\begin{array}{l}\text { ManagedJob findJobNamed(in string jobName) raises } \\
\text { (ManagedJobRetrievalFailedSignal); }\end{array}$ & $\begin{array}{l}\text { Returns a handle to the requested } \\
\text { job; raises ManagedJobRetrieval- } \\
\text { FailedSignal if not found. }\end{array}$ \\
\hline $\begin{array}{l}\text { ManagedJob findQueuedJobNamed(in string job- } \\
\text { Name) raises (ManagedJobRetrievalFailedSignal); }\end{array}$ & $\begin{array}{l}\text { Like findJobNamed, but restrict } \\
\text { search to jobs in the Queued or } \\
\text { Queued-Held states. }\end{array}$ \\
\hline $\begin{array}{l}\text { ManagedJob findActiveJobNamed(in string jobName) } \\
\text { raises (ManagedJobRetrievalFailedSignal); }\end{array}$ & $\begin{array}{l}\text { Like findJobNamed, but restrict } \\
\text { search to jobs in one of the following } \\
\text { states: Executing; Pausing; Paused; } \\
\text { Stopping; Aborting; Stopping- } \\
\text { Aborting; } \\
\text { Pausing-Aborting; Pausing-Stopping- } \\
\text { Aborting. }\end{array}$ \\
\hline ManagedJob findCompletedJobNamed(in string job- \\
Name) raises (ManagedJobRetrievalFailedSignal); & $\begin{array}{l}\text { Like findJobNamed, but restrict } \\
\text { search to jobs in the Completed state. }\end{array}$ \\
\hline ManagedJobSequence allQueuedManagedJobs(); & $\begin{array}{l}\text { Returns a sequence of all jobs in the } \\
\text { Queued or Queued-Held states. }\end{array}$ \\
\hline ManagedJobSequence allActiveManagedJobs(); & $\begin{array}{l}\text { Returns a sequence of all jobs in } \\
\text { one of the following states: Ex- } \\
\text { ecuting; Pausing; Paused; Stop- } \\
\text { ping; Aborting; Stopping-Aborting; } \\
\text { Pausing-Stopping; Pausing-Aborting; } \\
\text { Pausing-Stopping-Aborting. }\end{array}$ \\
\hline ManagedJobSequence allCompletedManagedJobs(); & $\begin{array}{l}\text { Returns a sequence of all jobs in one } \\
\text { of the following states: Completed; } \\
\text { Stopped; Aborted. }\end{array}$ \\
\hline $\begin{array}{l}\text { Returns a sequence of all jobs in the } \\
\text { Completed state. }\end{array}$ \\
\hline
\end{tabular}




\begin{tabular}{|l|l|}
\hline ManagedJobSequence allStoppedManagedJobs(); & $\begin{array}{l}\text { Returns a sequence of all jobs in the } \\
\text { Stopped state. }\end{array}$ \\
\hline ManagedJobSequence allAbortedManagedJobs(); & $\begin{array}{l}\text { Returns a sequence of all jobs in the } \\
\text { Aborted state. }\end{array}$ \\
\hline ManagedJobSequence allCancelledManagedJobs(); & $\begin{array}{l}\text { Returns a sequence of all jobs in the } \\
\text { Cancelled state. }\end{array}$ \\
\hline ManagedJobSequence allManagedJobs(); & $\begin{array}{l}\text { Returns a sequence of all jobs, ex- } \\
\text { cluding only those that have not yet } \\
\text { been started (i.e. in the Created or } \\
\text { Created-Held states). }\end{array}$ \\
\hline WorkcellJobManagerSequence allWorkcells(); & $\begin{array}{l}\text { Returns a sequence of all the work- } \\
\text { cells' job manager objects. }\end{array}$ \\
\hline
\end{tabular}

Table 2: Status operations available on all ManagedJobs

\begin{tabular}{|l|l|}
\hline \multicolumn{1}{|c|}{ Operation } & \multicolumn{1}{c|}{ Description } \\
\hline boolean isAborting(); & $\begin{array}{l}\text { True if job is Aborting, Pausing-Aborting, Stopping- } \\
\text { Aborting, or Pausing-Stopping-Aborting }\end{array}$ \\
\hline boolean isAborted(); & True if job is Aborted \\
\hline boolean isActive(); & $\begin{array}{l}\text { True if job is Executing, Pausing, Paused, Stopping, } \\
\text { Aborting, Stopping-Aborting, Pausing-Stopping, Pausing- } \\
\text { Aborting, or Pausing-Stopping-Aborting. }\end{array}$ \\
\hline boolean isCancelled(); & True if job is Cancelled. \\
\hline boolean isCompleted(); & True if job is Completed. \\
\hline boolean isCreated(); & True if job is Created or Created-Held. \\
\hline boolean isFinished(); & True if job is Completed, Stopped, or Aborted. \\
\hline boolean isPausing(); & $\begin{array}{l}\text { True if job is Pausing, Pausing-Stopping, Pausing- } \\
\text { Aborting, or Pausing-Stopping-Aborting. }\end{array}$ \\
\hline boolean isPaused(); & True if job is Paused, Created-Held, or Queued-Held. \\
\hline boolean isQueued(); & True if job is Queued or Queued-Held. \\
\hline boolean isStopping(); & $\begin{array}{l}\text { True if job is Stopping, Pausing-Stopping, Stopping- } \\
\text { Aborting, or Pausing-Stopping-Aborting. }\end{array}$ \\
\hline boolean isStopped(); & True if job is Stopped. \\
\hline attribute mj_state jobState; & Returns the state of the job as an enum. \\
\hline attribute string jobName; & The name of the job. \\
\hline
\end{tabular}

Table 3: Additional ShopJob status operations

\begin{tabular}{|l|l|}
\hline \multicolumn{1}{|c|}{ Operation } & \multicolumn{1}{c|}{ Description } \\
\hline attribute Lot theLot; & The Lot that was specified for the job. \\
\hline attribute short priority; & The priority that was specified for the job. \\
\hline attribute ManagedJobSequence Tasks; & $\begin{array}{l}\text { The sequence of ShopProcessJobs associated with } \\
\text { this job. }\end{array}$ \\
\hline
\end{tabular}

Table 4: Additional ShopProcessJob status operations

\begin{tabular}{|l|l|}
\hline \multicolumn{1}{|c|}{ Operation } & \multicolumn{1}{c|}{ Description } \\
\hline attribute WorkcellJob the_task; & $\begin{array}{l}\text { The WorkcellJob corresponding to } \\
\text { this ShopProcessJob. }\end{array}$ \\
\hline
\end{tabular}




\begin{tabular}{|l|l|}
\hline attribute WorkcellJobManager workcell; & $\begin{array}{l}\text { The workcell to which this task is cur- } \\
\text { rently assigned (null if not assigned). }\end{array}$ \\
\hline attribute ShopJob parentjob; & $\begin{array}{l}\text { The ManagedJob that contains this } \\
\text { ShopProcessJob. }\end{array}$ \\
\hline $\begin{array}{l}\text { attribute DocumentSpecification ProcessOperation- } \\
\text { DocumentRef; }\end{array}$ & $\begin{array}{l}\text { The document ref from the routing } \\
\text { for this task. }\end{array}$ \\
\hline attribute IdentifierSequence EligibleWorkcells; & $\begin{array}{l}\text { Sequence of IDs of eligible workcells } \\
\text { copied from the routing. }\end{array}$ \\
\hline attribute Duration routingexpectedtimetocomplete; & $\begin{array}{l}\text { Expected time to complete copied } \\
\text { from the routing. }\end{array}$ \\
\hline
\end{tabular}

\subsection{Commands}

The Executor also provides operations that a client (i.e. the Guardian) can use to control both the state of shop-level jobs and the state of the Executor itself. The control functions are divided into three categories: the functions to control the Executor (Table 5), the functions to control single jobs (Table 6), and the functions to control all jobs (Table 7). The "all" functions simply broadcast one of the single-job functions to every job known to the Executor.

All of the operations described in this section are provided by the JobManager class, not by the ManagedJob class.

Table 5: Commands affecting the state of the Executor

\begin{tabular}{|l|l|}
\hline \multicolumn{1}{|c|}{ Operation } & \multicolumn{1}{c|}{ Description } \\
\hline oneway void abortManagerOperations(); & $\begin{array}{l}\text { Causes the Executor to emergency-stop and cease } \\
\text { to exist. }\end{array}$ \\
\hline void pauseManagerOperations(); & $\begin{array}{l}\text { Causes the Executor to enter the Pausing state } \\
\text { and stop dispatching new shop-level jobs onto the } \\
\text { shop floor. When the shop floor is idle, the Ex- } \\
\text { ecutor enters the Paused state. New jobs will still } \\
\text { be accepted and placed in the queue. }\end{array}$ \\
\hline void stopManagerOperations(); & $\begin{array}{l}\text { Causes the Executor to enter the Stopping state } \\
\text { and stop dispatching new shop-level jobs onto the } \\
\text { shop floor. When the shop floor is idle, the Execu- } \\
\text { tor enters the Stopped state. New jobs will NOT } \\
\text { be accepted. }\end{array}$ \\
\hline void resumeManagerOperations(); & $\begin{array}{l}\text { Causes the Executor to return to the Executing } \\
\text { or Waiting state from Pausing, Stopping, Pausing- } \\
\text { Stopping, Paused, or Stopped. }\end{array}$ \\
\hline
\end{tabular}

Table 6: Commands affecting one shop-level job

\begin{tabular}{|l|l|}
\hline \multicolumn{1}{|c|}{ Operation } & \multicolumn{1}{c|}{ Description } \\
\hline ManagedJob createJob(); & $\begin{array}{l}\text { Returns a handle to a newly created, } \\
\text { uninitialized job. The caller should } \\
\text { initialize the job and then pass it to } \\
\text { startJob. }\end{array}$ \\
\hline
\end{tabular}




\begin{tabular}{|l|l|}
\hline $\begin{array}{l}\text { void startJob (in ManagedJob aManagedJob) raises } \\
\text { (ManagedJobNotAcceptedSignal); }\end{array}$ & $\begin{array}{l}\text { Place a new job into the queue so } \\
\text { that the Executor will dispatch it. } \\
\text { Raises ManagedJobNotAcceptedSig- } \\
\text { nal if the Executor is Stopping or } \\
\text { Stopped, or if the job has a problem } \\
\text { like duplicate name or invalid lot. }\end{array}$ \\
\hline $\begin{array}{l}\text { void pauseJob (in ManagedJob aManagedJob) raises } \\
\text { (InvalidStateTransitionSignal); }\end{array}$ & $\begin{array}{l}\text { Causes the job to enter the Pausing } \\
\text { state and stop dispatching new tasks } \\
\text { to workcells. When all running tasks } \\
\text { have completed, the job enters the } \\
\text { Paused state and remains in the ac- } \\
\text { tive list. }\end{array}$ \\
\hline $\begin{array}{l}\text { void stopJob (in ManagedJob aManagedJob) raises } \\
\text { (InvalidStateTransitionSignal); }\end{array}$ & $\begin{array}{l}\text { Analogous to pauseJob, except that } \\
\text { when all running tasks have com- } \\
\text { pleted, the job enters the Stopped } \\
\text { state and is considered finished. A } \\
\text { stopJob directed at a job that is } \\
\text { merely Queued will cause it to be } \\
\text { Cancelled. }\end{array}$ \\
\hline $\begin{array}{l}\text { Causes a paused job to return to the } \\
\text { (InvalidStateTransitionSignal); }\end{array}$ \\
\hline $\begin{array}{l}\text { void abortJob (in ManagedJob aManagedJob) raises } \\
\text { (InvalidStateTransitionSignal); }\end{array}$ & $\begin{array}{l}\text { Causes a job to enter the Abort- } \\
\text { ing state and cascade aborts to any } \\
\text { currently executing tasks. When all } \\
\text { tasks have aborted, the job enters the } \\
\text { Aborted state and is considered fin- } \\
\text { ished. An abortJob directed at a job } \\
\text { that is merely Queued will cause it to } \\
\text { be Cancelled. }\end{array}$ \\
\hline Job);
\end{tabular}

Table 7: Commands affecting all shop-level jobs

\begin{tabular}{|l|l|}
\hline \multicolumn{1}{|c|}{ Operation } & \multicolumn{1}{|c|}{ Description } \\
\hline void makeAllManagedJobsPausing(); & $\begin{array}{l}\text { Effectively issues a pauseJob for each job. Excep- } \\
\text { tions are not propagated. }\end{array}$ \\
\hline void makeAllManagedJobsNotPaused(); & $\begin{array}{l}\text { Effectively issues a resumeJob for each job. Ex- } \\
\text { ceptions are not propagated. }\end{array}$ \\
\hline void makeAllManagedJobsAborting(); & $\begin{array}{l}\text { Effectively issues an abortJob for each job. Ex- } \\
\text { ceptions are not propagated. }\end{array}$ \\
\hline void makeAllManagedJobsStopping(); & $\begin{array}{l}\text { Effectively issues a stopJob for each job. Excep- } \\
\text { tions are not propagated. }\end{array}$ \\
\hline
\end{tabular}




\subsection{Initialized ShopJob fields}

When the Guardian wants to start a new shop-level job, it must call createJob, initialize three fields in the job object that is returned, and then call startJob. The three fields are:

1. attribute string jobName;

The name of the job. Must be unique.

2. attribute Lot theLot;

Must be a valid key in the PIB to retrieve the lot from which the routing spec is retrieved.

3. attribute short priority;

Optional. Controls the order in which queued shop jobs are dispatched onto the shop floor. 0 is the default; lower numbers cause jobs to be dispatched more quickly. (This is analogous to Unix process priorities.)

\subsection{State diagrams}

The state sets used by JobManager and ManagedJob are flattened implementations of concurrent state sets ${ }^{1}$ derived from the CIMF (CIM Framework). Figure 4 shows the states of the JobManager class which the Executor implements. The Executor starts up in the Initializing state, then enters the Waiting state when it is ready to accept jobs. When it is given work to do, it enters the Executing state and only returns to the Waiting state when it is again idle with no jobs in its queue or active list.

Once it has received an abortManagerOperations message, the Executor irrevocably heads for the "aborted" state. ("Aborted" is not really a state; it represents the Executor having terminated.)

Figure 5 shows the states of the ManagedJob class which are used by ShopJob and ShopProcessJob. While a JobManager has only one terminal state, ManagedJobs can end up Completed, Stopped, Aborted, or Cancelled. A Cancelled job is one that was stopped or aborted before work on it even began. Unlike the analogous stopManagerOperations, a stopJob command is just as irrevocable as an abortJob. It is possible to throw a Stopping job into the Aborted state with an abortJob, but it is not possible to resume it.

As can be seen from reading the descriptions of the various state-changing commands in Tables 5 through 7 , pausing or stopping involves reaching a convenient pause-point or stop-point. The JobManager does not pause or stop while there are active shop jobs; neither does a shop job pause or stop while there are active tasks. Instead, they refrain from starting any new work until the running jobs or tasks have completed, and then enter the Paused or Stopped state. Aborts, on the other hand, have no preconditions. When it is told to abort, the Executor will terminate immediately, without waiting for workcell activity to subside. A shop job that is aborted will cascade aborts to any active workcell tasks so that activity on the shop job will cease immediately. It is then left for the operator to clean up any problems that result.

\section{$3 \quad$ Issues for Future Work}

The preceding represents the interface of the Executor as it was used for the 1996 NAMT Framework demo. This was only the first year of a five-year project; the Executor is expected to continue to evolve in response to lessons learned, better understanding of the specifications, and improvements to the infrastructure. In support of that evolution, commentary on the limitations and possible problems with the 1996 interface is presented here.

\footnotetext{
${ }^{1}$ In a "concurrent" state model, the state of a machine is modeled using two or moze independent state variables. For example, the state of a two-engine airplane may be modeled using independent state variables for each engine.
} 


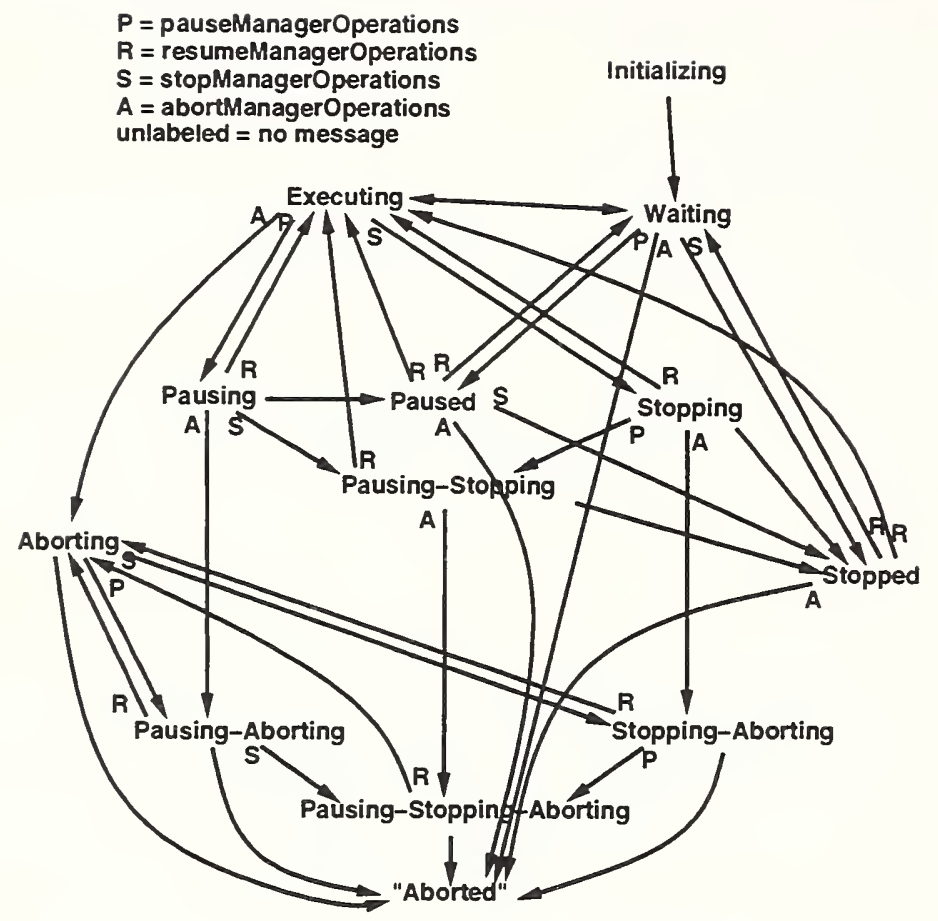

Figure 4: JobManager states

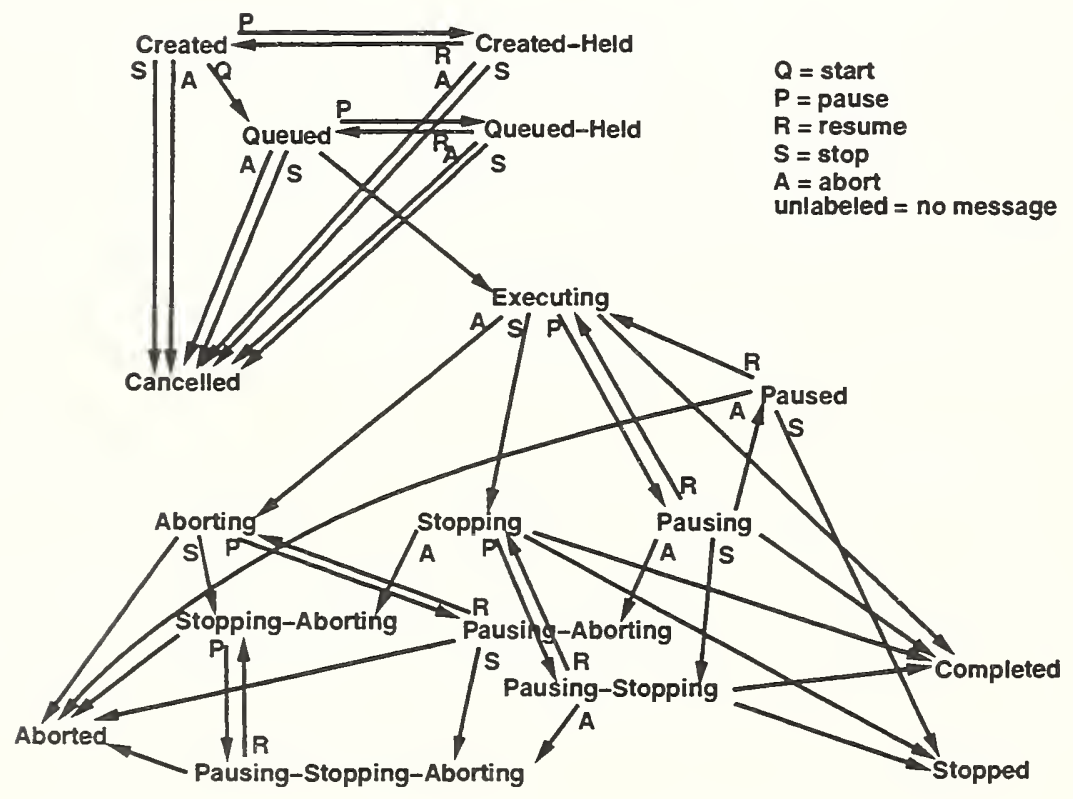

Figure 5: ManagedJob states 


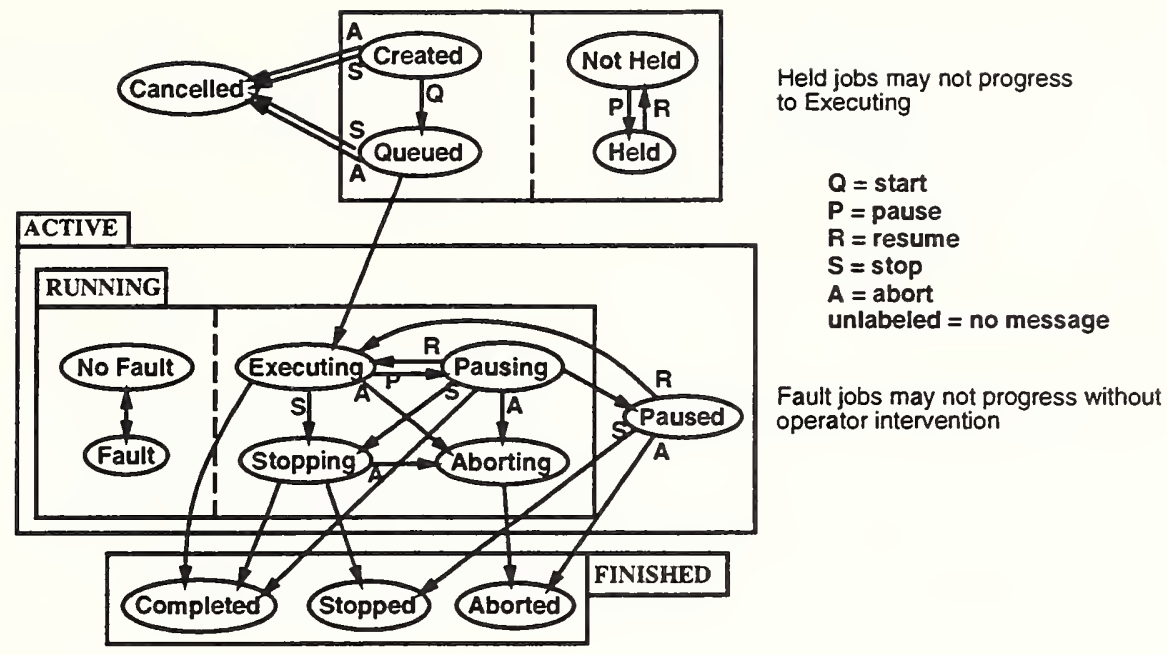

Figure 6: Proposed ManagedJob states (concurrent model)

\subsection{States}

The concurrent states model of the CIMF has been the subject of controversy and misunderstanding. Having discussed the issue with Ed Barkmeyer, another member of the Framework team, we now believe that the concurrency as implemented is not needed, and that another form of concurrency is needed but lacking.

The combinations of Pausing-Stopping, Pausing-Aborting, Stopping-Aborting, and PausingStopping-Aborting are not meaningful to us. Aborts take precedence over stops, and stops take precedence over pauses. The notion that a job can pause in the middle of a stop or abort - to "freeze" immediately, then resume and continue on its way to the stop point or abort point - is not valid. If a machine is in the process of cutting metal, any kind of "freeze" is equivalent to an emergency stop, with ramifications even more serious than those of an abort.

Instead of this invalid concurrency, what is needed is a way to represent fault states. For example, if a workcell has been told to stop, but before it can reach a stop-point a machine gets stuck and cannot proceed, the state of the task is modeled by Stopping-Fault. The Executing, Pausing, Paused, Stopping, and Aborting states should all be mutually exclusive, but they may all be concurrent with the Fault state. That way, it is clear when human intervention is needed, and a task that is in a fault state can be recovered and continued after an operator has intervened. Figure 6 illustrates this proposed model.

\subsection{Job control commands}

In the interface described by this report, job control commands that affect a particular job are part of the JobManager interface and contain an argument specifying which job to affect. Since the state of the job can only be queried through the ManagedJob interface, it would make more sense for the state of the job to be controlled via the ManagedJob interface. That is the way it was organized in CIMF 1.3.

In the model underlying such an organization, the interface to a JobManager and the interfaces to related ManagedJobs are different aspects of a single agent. The JobManager provides access to services for controlling and monitoring the operation of the combined agent as well as some job control functions that can be applied to the aggregation of its related ManagedJobs. The ManagedJob interface itself provides for job control and monitoring of individual jobs. These interfaces are very similar in function to the Administrative and Task components of the Job Controller defined in the Manufacturing System Integration (MSI) project's Control Entity Interface (CEI).[3] What could not easily be done in the CEI, since an object oriented communications infrastructure was not available, was to make the tasks (jobs) directly addressable. But with CORBA, operations can be 
defined directly on the job (task) interface.

The existing job control interface, a mixture of CIMF 1.3 and CIMF 1.2, was used because we could not find a working interpretation of CIMF 1.3. SEMATECH has since explained how they intended CIMF 1.3 to work, so the NAMT Framework testbed could and should be migrated in that direction in future development.

\section{Further Reading}

This is one of a number of documents that are to be written about the 1996 NAMT Framework project. Most significantly, a Framework Compendium is planned to provide an overview of the entire project, and a report will be produced that summarizes the results for the validation of the CIM Framework and SEMATECH's response. Currently the process of exchanging results with SEMATECH is still ongoing, and the other documents are not yet finalized.

Related reports will also be available on NIST's external and internal web servers from <URL:http://www.nist.gov/framework/> and <URL:http://www-i.cme.nist.gov/framework/doc /papers96/> respectively.

\section{References}

[1] Howard M. Bloom and Neil Christopher. A framework for distributed and virtual discrete part manufacturing. In Proceedings of the CALS EXPO '96, Long Beach, CA, October 1996.

[2] Lawrence Eng, Ken Freed, Jim Hollister, Carla Jobe, Paul McGuire, Alan Moser, Vinayak Parikh, Margaret Pratt, Fred Waskiewicz, and Frank Yeager. Computer Integrated Manufacturing (CIM) Application Framework Specification 1.3. SEMATECH, 2706 Montopolis Drive, Austin, TX 78741 U.S.A., 1996.

[3] Sarah Wallace, M. K. Senehi, Ed Barkmeyer, Steven Ray, and Evan K. Wallace. Manufacturing Systems Integration: Control Entity Interface Specification. National Institute of Standards and Technology, Interagency Report 5272, 1993. Available from the National Technical Information Service, Springfield, VA 22161 U.S.A. 


\section{A job_mgr.idl}

// Interface definition for generic job and job manager components of the NAMT

// Framework

// Started: 1996-07-30 by Dave Flater

// Status: Fairly stable

// The following definitions were originally from:

// Part 1: IDL from CIM Framework, specifically:

// Modified IDL from CIM Framework Spec 1.3

// Updated by Paul McGuire, SEMATECH, March 29, 1996

// Many things have been modified for any or all of the following

// reasons:

//

$/ /$ 1. Working around inconsistencies in the CIMF;

$/ /$ 2. Correcting obvious errors in the CIMF;

// 3. Reducing complexity for the trial implementation;

// 4. Making NAMT components play together.

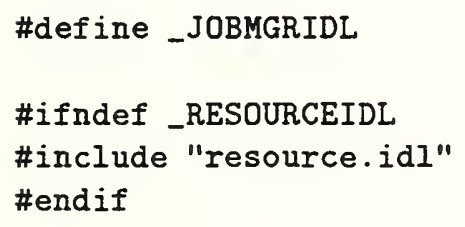

\#define _JOBMGRIDL

\#ifndef_RESOURCEIDL

\#include "resource.idl"

\#endif

interface ManagedJob;

\#ifndef_MJS

typedef sequence<ManagedJob> ManagedJobSequence;

\#define_MJS

\#endif

\section{// ManagedJob states.}

// Flattened implementation of the concurrent state combinations from

// p. 41 of the CIMF. Technically, any active state is "EXECUTING,"

// but here MJ_EXECUTING means very specifically the state in which

// we are active, not pausing, not stopping, and not aborting.

//

// Created-held and queued-held have been added

// because a makeAllManagedJobsPausing probably wants to "hold"

$/ /$ any queued jobs even if a workcell becomes available. Still

// lacking is a fault state, as described by these comments quoted

// from executor_i.cc:

// If one of my tasks was stopped or aborted, but the ShopJob was

// running normally, then I have a problem. In the MSI model, the

// ShopJob enters a fault state and a red light flashes on the

$/ /$ console to get a human to do something. The situation is

// resolved when the human sends something like Abort, Retry, Call

// Complete, or Defer. Maybe as future work we will add this to

// the CIMF, but currently all we can do is cascade the stop or

$/ /$ abort upward. 
// Also possible is future addition of MJ_PAUSED_STOPPING,

// MJ_PAUSED_ABORTING, and MJ_PAUSED_STOPPINGABORTING to model various

// strange fault states.

enum $m j_{\text {_state }}$ \{MJ_CREATED, MJ_CREATED_HELD,

MJ_QUEUED, MJ_QUEUED_HELD,

MJ_EXECUTING, MJ_PAUSING_EXECUTION, MJ_PAUSED_EXECUTION,

MJ_STOPPING, MJ_ABORTING, MJ_STOPPINGABORTING,

MJ_PAUSINGSTOPPING, MJ_PAUSINGABORTING ,

MJ_PAUSINGSTOPPINGABORTING,

MJ_STOPPED, MJ_CANCELLED, MJ_ABORTED, MJ_COMPLETED\};

// JobManager states. These are based loosely on the

// MachineResource class of the CIMF (p. 223). JobManager and

// ProcessJobManager don't seem to have state diagrams.

// Note that CIMF MachineResource doesn't admit to a STOPPED state.

enum $j m_{-}$state \{JM_INITIALIZING, JM_WAITING, JM_EXECUTING, JM_PAUSING_EXECUTION, JM_PAUSED_EXECUTION, JM_STOPPING, JM_ABORTING, JM_STOPPINGABORTING, JM_PAUSINGSTOPPING, JM_PAUSINGABORTING, JM_PAUSINGSTOPPINGABORTING, JM_STOPPED\};

// Generic JobManager to be inherited by ShopJobManager and WorkcellJobManager.

interface JobManager

\{

// EXCEPTIONS

/* The search operation for a job failed. */

exception ManagedJobRetrievalFailedSignal \{\};

/* Attempt to create or start a new job rejected. This will happen

if the JobManager is in the JM_STOPPED state. */

exception ManagedJobNotAcceptedSignal \{\};

l*

OPERATIONS FOR TOP-DOWN CONTROL AND MONITORING

These are taken from JobManager. MachineResource comes with operations like shutdownNormal and shutdownImmediate that would be redundant here.

Helpful hints:

1. abortManagerOperations means E-Stop. The JobManager goes away

2. pauseManagerOperations and stopManagerOperations both mean that you should let any running jobs finish but don't start any new ones. The only difference between STOPPED and PAUSED is that you can queue up new jobs while PAUSED but not while STOPPED. 
3. You can either stop or abort a job that is merely created or queued to send it to the MJ_CANCELLED state.

$* /$

// COMMANDS AFFECTING THE STATE OF THE JOB MANAGER

oneway void abortManageroperations();

void pauseManagerOperations();

void resumeManageroperations();

void stopManagerOperations();

// COMMANDS AFFECTING THE STATE OF ALL MANAGED JOBS

void makeAllManagedJobsPausing();

void makeAllManagedJobsNotPaused ();

void makeAllManagedJobsAborting ();

void makeAllManagedJobsStopping();

// COMMANDS AFFECTING THE STATE OF ONE MANAGED JOB (CIMF 1.2)

// queuejob has been removed; just use startJob.

ManagedJob createJob();

void startJob (in ManagedJob aManagedJob);

void pauseJob (in ManagedJob aManagedJob);

void resumeJob (in ManagedJob aManagedJob);

void abortJob (in ManagedJob aManagedJob);

void stopJob (in ManagedJob aManagedJob);

// OTHER ADMINISTRATIVE OPERATIONS

void removeFinishedJob(in ManagedJob aManagedJob);

// OPERATIONS TO QUERY JOB MANAGER STATE

boolean isPaused();

jm_state jobManagerState();

// OPERATIONS TO ACCESS MANAGED JOBS

ManagedJob findJobNamed(in string jobName)

raises (ManagedJobRetrievalFailedSignal);

ManagedJob findQueuedJobNamed(in string jobName)

raises (ManagedJobRetrievalFailedSignal);

ManagedJob findActiveJobNamed (in string jobName)

raises (ManagedJobRetrievalFailedSignal);

ManagedJob findCompletedJobNamed(in string jobName)

raises (ManagedJobRetrievalFailedSignal);

ManagedJobSequence allQueuedManagedJobs ();

ManagedJobSequence allActiveManagedJobs ();

ManagedJobSequence allFinishedManagedJobs ();

ManagedJobSequence all CompletedManagedJobs();

ManagedJobSequence allStoppedManagedJobs();

ManagedJobSequence allAbortedManagedJobs();

ManagedJobSequence allCancelledManagedJobs();

ManagedJobSequence allManagedJobs();

// OPERATIONS FOR BOTTOM-UP REPORTING

// Task name instead of a ManagedJob handle is used here due to 


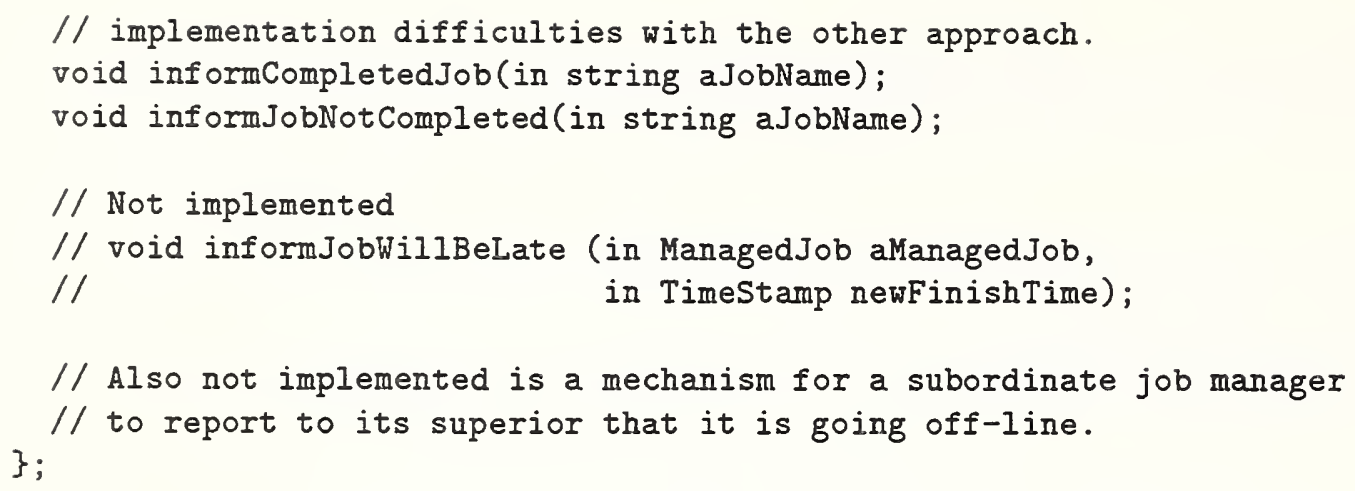

\section{// ATTRIBUTES}

attribute mj_state jobstate;

// This attribute is called jobName instead of just name because there // was a conflict with some internal Orb function also called name. attribute string jobName;

// Any job except the lowest-level job is comprised of a sequence of // "tasks" or "operations." This attribute provides a place to // store and access that sequence. attribute ManagedJobSequence Tasks;

// Priority queues may or may not be supported. attribute short priority; // Like Unix, 0 = default \}; 


\section{B executor.idl}

// Interface definition for the Executor component of the NAMT

// Framework

// Started: 1996-06-03 by Dave Flater

// Major revision: 1996-07-30

// Status: Fairly stable

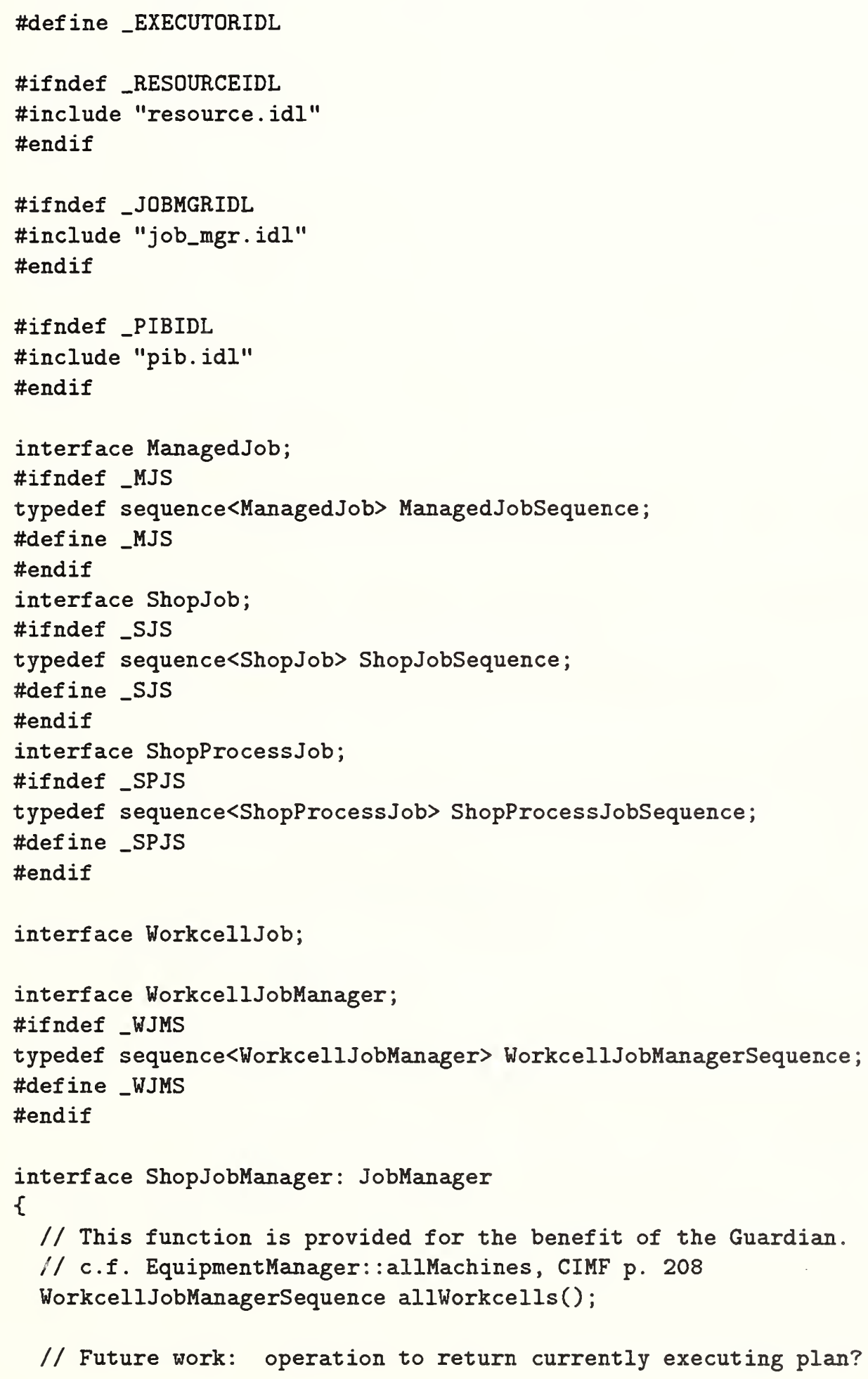




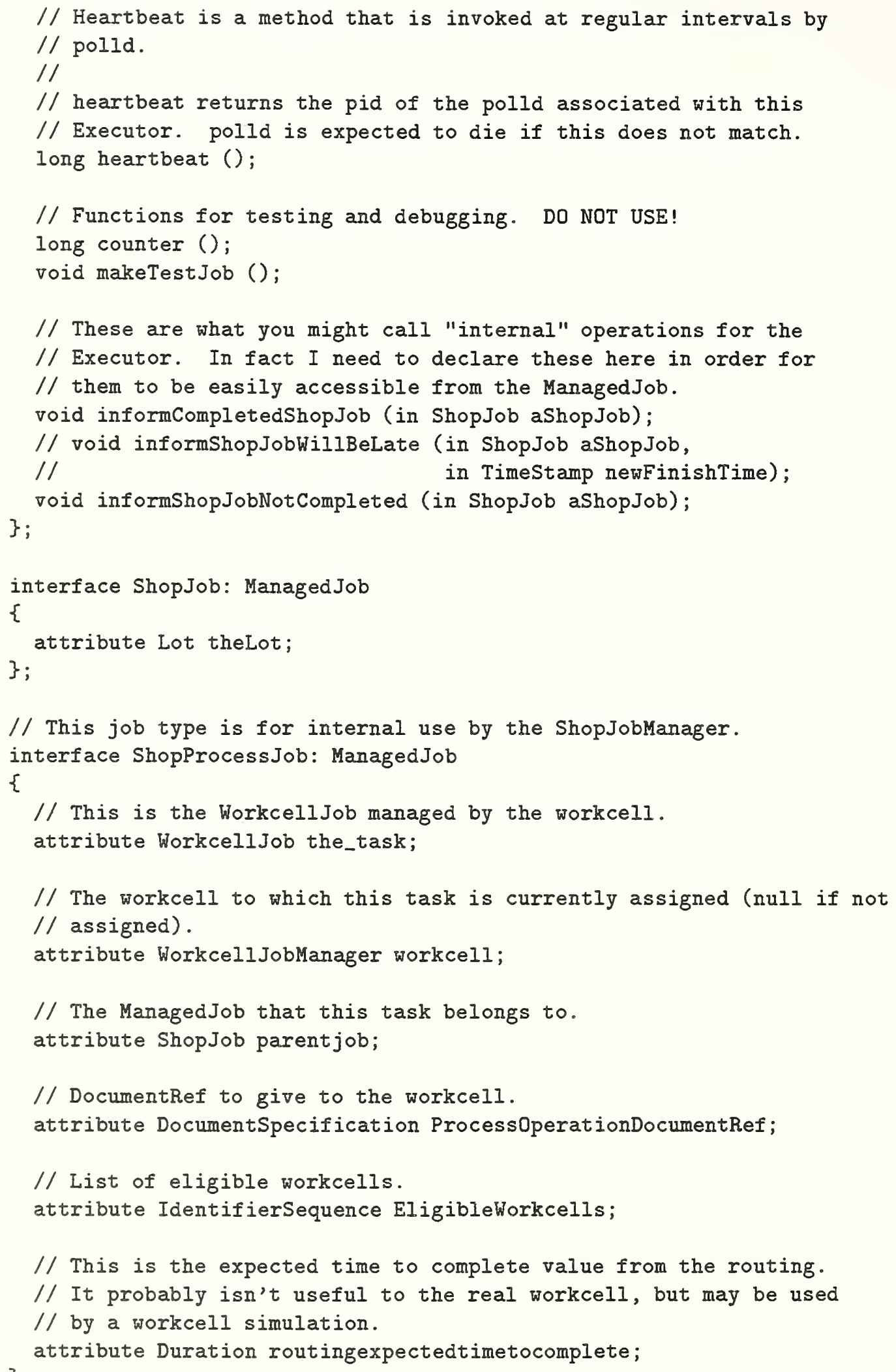




\section{C workcell.idl}

// Interface definition for Workcell

// Started: 1996-06-03 by Dave Flater

// Major revision: 1996-07-08

// Status: Semi-stable

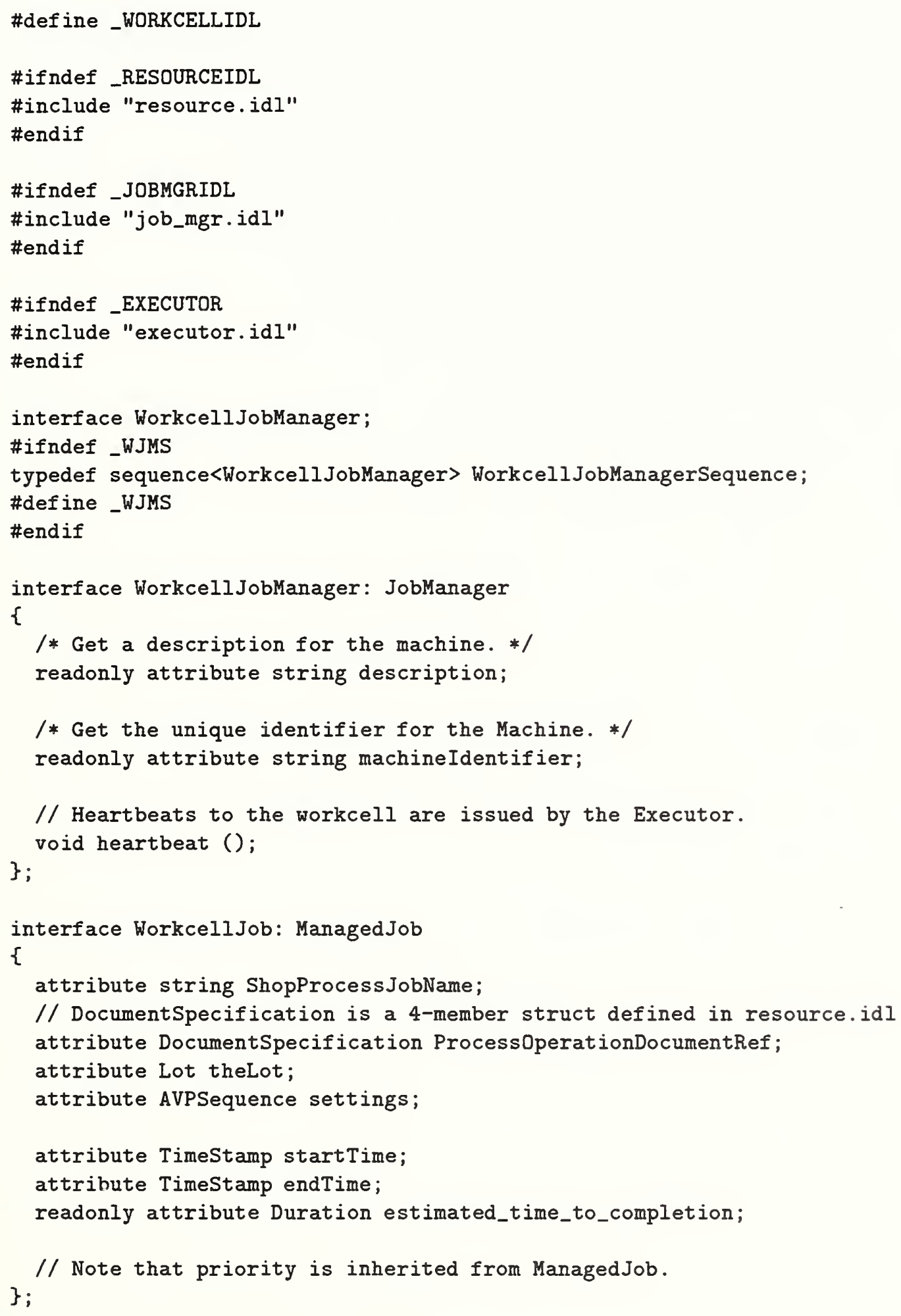



